\title{
Sub-degree CMB anisotropy from space. I. Sky coverage and sensitivity
}

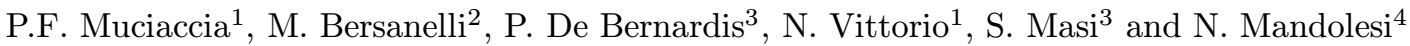 \\ 1 Dipartimento di Fisica, Universita' di Tor Vergata, Roma, Italy \\ 2 Istituto di Fisica Cosmica, CNR, Milano, Italy \\ 3 Dipartimento di Fisica, Universita' La Sapienza, Roma, Italy \\ 4 Istituto TESRE, CNR, Bologna, Italy
}

Received August 7; accepted November 27, 1995

\begin{abstract}
Decisive progress in cosmology is expected from a space mission dedicated to an extensive mapping of the Cosmic Microwave Background (CMB) with high angular and temperature sensitivity. Based on the European Space Agency COBRAS/SAMBA mission concept, we have studied the attainable sensitivity and sky coverage taking into account the effect of solar-system sources for the two far-Earth candidate orbits (Lagrangian points Moon-Earth L5 and Sun-Earth L2). We also take into account the microwave emission of the Galaxy in limiting the useful sky coverage. Our results provide a quantitative assessment of the relative observational merits of the two orbit options, and show that the Sun-Earth L2 orbit allows significantly better observational conditions.
\end{abstract}

Key words: cosmic microwave background — space vehicles — cosmology: observations

\section{Introduction}

Accurate measurements of the Cosmic Microwave Background ( $\mathrm{CMB}$ ) anisotropy provide a unique probe to investigate the physical conditions in the early universe and are emerging as one of the most powerful tools to discriminate among different cosmological and structureformation models (e.g. Vittorio et al. 1991; Bennett et al. 1992; Crittenden et al. 1993; Bond et al. 1994; Kamionkowsky et al. 1994; Coulson et al. 1994, for a review see White et al. 1994). Following the first detection of large $\left(>7^{\circ}\right)$ angular scale anisotropy by the COBEDMR team (Smoot et al. 1992), a number of balloonborne and ground-based experiments have reported detection at comparable $\Delta T / T$ levels. At the COBE angular scale the $\Delta T / T$ distribution traces the primordial density perturbations, while scales below $\sim 1^{\circ}$ are sensitive to the assumed cosmological model and to the relative abundance of baryonic and non baryonic dark matter. Most of the recent effort has concentrated in the sub-degree range, aiming to a measurement of the power spectrum of the CMB fluctuations at high-order multipoles (e.g. Schuster et al. 1993; Wollack et al. 1993; Cheng et al. 1994; De Bernardis et al. 1994; Dragovan et al. 1994; Devlin et al. 1994; Clapp et al. 1994; Gundersen et al. 1995; Netterfield et al. 1995). Theory anticipates rms temperature fluctua-

Send offprint requests to: P.F. Muciaccia tions $\sqrt{C(0)}$ of the order of $\lesssim 50-100 \mu \mathrm{K}$ when observed through a 30 arcmin FWHM beam, the exact rms value depending on the specific cosmological model.

While ground-based and balloon-borne experiments provide valuable results, it appears that only a dedicated space mission will be able to map the CMB anisotropy with sufficient sky coverage, sensitivity and spectral range to produce definitive observations of the CMB anisotropies at sub-degree scales (see Danese et al. 1996 for a recent discussion). Present sub-orbital experiments are limited by atmospheric noise (Church 1995; Bersanelli et al. 1995a) and astrophysical foregrounds, and suffer a very limited sky coverage. A space mission, besides overcoming the problem of atmospheric disturbance, will ensure the large sky coverage, spectral range and integration time required for a better understanding of the foreground components, as well as other potential systematic effects. An extended, high-resolution imaging of the CMB spatial distribution will allow an accurate reconstruction of the power spectrum of the primordial fluctuations, with an outstanding scientific return for cosmology astro-particle physics (e.g. Readhead \& Lawrence 1992; White et al. 1994; Danese et al. 1996, and references therein).

At present, a number of European and US space projects have been proposed, or are under study, to address this fundamental observational goal. One of them, COBRAS/SAMBA, is a European Space Agency 
candidate for the M3 mission, and is currently undergoing the feasibility (Phase $A$ ) study. To optimize the quality of the observations a number of critical trade-offs must be faced between cost, orbit, scanning technique, sensitivity, sky coverage, acceptable off-axis signals, thermal stability, etc. In particular, depending on the selected orbit and scanning technique, the effect of local, large-solidangle sources (typically the Sun, Galaxy, Earth, Moon and planets) must be studied in conjunction with the expected instrumental performances in order to evaluate possible solutions. In this paper we report the results of accurate simulations of the expected observational capability of the COBRAS/SAMBA Low Frequency Instrument for the two far-Earth orbits presently considered, including the effects of solar system sources and the foreground emission of our Galaxy.

\section{COBRAS/SAMBA: Instrument and observing strategy}

The COBRAS/SAMBA mission has been conceived to provide high-sensitivity $(\Delta T \lesssim 10 \mu \mathrm{K})$, high-resolution $(\lesssim$ $30^{\prime}$ FWHM), near-full-sky maps of the CMB anisotropies, with a high level of control over foreground diffuse emissions and systematic errors (Mandolesi et al. 1993; Puget et al. 1993; Mandolesi et al. 1995a). The model payload ${ }^{1}$ consists mainly of a Gregorian telescope, with a $1.5-\mathrm{m}$ parabolic primary reflector and a secondary mirror, leading to an integrated focal plane instrument. The telescope is surrounded by a flared shield to improve rejection of off-axis radiation and stray light. In order to cover the wide frequency range that will enable accurate separation of the foreground sources from the cosmic signal two different types of detectors will be used. The focal plane instrument will incorporate eight frequency bands provided by four arrays of cryogenically-cooled bolometers in the range 140-800 GHz (High-Frequency Instrument, HFI), and four arrays of passively-cooled, low-noise radiometers in the range $30-130 \mathrm{GHz}$ (Low-Frequency Instrument, LFI). Table 1 summarizes the main characteristics of the COBRAS/SAMBA payload.

A number of non-cosmological diffuse emissions (mainly synchrotron and free-free at low frequencies, and interstellar dust components at high frequencies; e.g. Toffolatti et al. 1995; Brandt et al. 1994) will affect the signal of the various bands in different proportions. The spectral information contained in the multifrequency maps, together with the large sky coverage, will allow strong control over residual foreground contamination and systematic errors.

\footnotetext{
${ }^{1}$ This work is based on the mission and instrument parameters as they emerged from the Assessment Study Phase. By the time this paper has been submitted and published, the project design has of course evolved somewhat. However, the main results are still valid, and were used in the Phase A trade-offs for orbit choice.
}

\subsection{Orbit and scanning technique}

Residual pick-up of Earth emission through the sidelobes of the optical system is one of the most worrying potential systematic effects in sub-orbital CMB experiments (e.g. Lubin 1995). A space mission carried out from a low-earth orbit (LEO) would not substantially improve the situation in this respect, since, for example, the Earth solid angle as seen from a $900 \mathrm{~km}$ orbit is $\sim 86 \%$ of what one gets in a ground-based experiment. The impact of this potential effect has to be compared to the goal sensitivity and is roughly inversely proportional to the antenna solid angle. An experiment with the COBRAS/SAMBA sensitivity and angular resolution carried out from the COBE-DMR orbit would require a rejection $10^{3}$ to $10^{4}$ times greater to keep the same contamination level of COBE-DMR. While a LEO could be studied for a sub-degree anisotropy smallmission, this option would require restrictive compromises with the scanning technique (affecting the achievable angular information) and the sky coverage.

A far-Earth orbit is required to minimize the effect of Earth radiation (e.g. Mandolesi et al. 1995b; Smoot et al. 1995), and improves the stability and absolute level of the local temperature, which are critical for both actively and passively cooled detectors. Within the COBRAS/SAMBA feasibility study two possible far-earth orbits have been selected and are currently under development: Earth-Moon L5 (hereafter EM-L5), the triangular Lagrangian point of the Earth-Moon system, at a distance of $400.000 \mathrm{~km}$; and a Lissajous or halo orbit around Sun-Earth L2 Lagrangian point (SE-L2), at a distance of $\sim 1.500 .000 \mathrm{~km}$ (see, e.g., Farquhar \& Dunham 1990). Figure 1 shows schematically such orbits compared to the Earth-Moon system.

The spacecraft will be spin-stabilized, with a spin rate of $1 \mathrm{rpm}$. For both the EM-L5 and SE-L2 options, thermal and radiometric considerations require the space craft orientation to be anti-sun during normal observation mode. In principle, a $\pm 15^{\circ}$ maneuver margin of the spin axis around the anti-sun orientation is allowed. The instrument field of view is offset by an angle $\alpha=70^{\circ}$ from the spin axis. This means that in every spin rotation the instruments will observe a circle in the sky, $140^{\circ}$ wide, centered in the spin axis direction (see Fig. 1). The baseline observing strategy (for both the EM-L5 and SE-L2 orbits) is to have the spin axis fixed in the anti-sun direction for 2 hours (or 120 spin rotations), and then move the spin direction by 5 arcmin along the ecliptical plane. This will compensate for the Earth revolution angle and will provide redundant coverage of the sky throughout the mission lifetime (2 to 5 years). With this mapping scheme, the two ecliptic polar caps (20 degrees around the poles) are not observed. Either a larger offset angle $\alpha$, or a periodic re-pointing of the spin axis (within $\pm 15^{\circ}$ ) can be used to optimize the sensitivity contrast and reach complete sky coverage. For the purpose of our simulations we have 
Table 1. COBRAS/SAMBA payload characteristics

\begin{tabular}{|c|c|c|c|c|c|c|c|c|}
\hline Telescope & \multicolumn{8}{|c|}{$\begin{array}{l}1.5 \mathrm{~m} \text { Diam. Gregorian; system emissivity } 1 \% \\
\text { Viewing direction offset } 30^{\circ} \text { from spin axis }\end{array}$} \\
\hline Instrument & \multicolumn{4}{|c|}{ LFI } & \multicolumn{4}{|c|}{ HFI } \\
\hline Center Frequency $(\mathrm{GHz})$ & 31.5 & 53 & 90 & 125 & 140 & 222 & 400 & 714 \\
\hline Wavelength (mm) & 9.5 & 5.7 & 3.3 & 2.4 & 2.1 & 1.4 & 0.7 & 0.42 \\
\hline Bandwidth $\left(\frac{\Delta \nu}{\nu}\right)$ & 0.15 & 0.15 & 0.15 & 0.15 & 0.4 & 0.5 & 0.7 & 0.6 \\
\hline Detector Technology & \multicolumn{4}{|c|}{ HEMT receiver arrays } & \multicolumn{4}{|c|}{ Bolometers arrays } \\
\hline Detector Temperature & \multicolumn{4}{|c|}{$\sim 100 \mathrm{~K}$} & \multicolumn{4}{|c|}{$0.1-0.15 \mathrm{~K}$} \\
\hline Cooling Requirements & \multicolumn{4}{|c|}{ Passive } & \multicolumn{4}{|c|}{ Cryocooler + Dilution system } \\
\hline Number of Detectors & 4 & 14 & 26 & 12 & 8 & 11 & 16 & 16 \\
\hline Angular Resolution (arcmin) & 30 & 30 & 30 & 30 & 10.5 & 7.5 & 4.5 & 3 \\
\hline Optical Transmission & 1 & 1 & 1 & 1 & 0.3 & 0.3 & 0.3 & 0.3 \\
\hline $\begin{array}{l}\text { Noise figures } \\
\text { LFI }\left(T_{\text {sys }}, \mathrm{K}\right) \\
\text { HFI }\left(\mathrm{NEP}, 10^{-17} \mathrm{~W} \mathrm{~Hz}^{-1 / 2}\right)\end{array}$ & 30 & 40 & 80 & 135 & 10.3 & 5.3 & 2.7 & 2.6 \\
\hline
\end{tabular}

Fig. 1. Schematic of the five Earth-Moon Lagrangian points and Sun-Earth L2. The satellite is shown in the SE-L2 orbit. The $70^{\circ}$ off-axis field of view scans the sky at a spin rate of $1 \mathrm{rpm}$, while the spacecraft is constantly in near-antisun direction

assumed the baseline $\pm 70^{\circ}$ offset angle with no additional maneuvers.

\subsection{Concept and performance of the low-frequency instrument}

The baseline LFI array consists of 28 corrugated feed horns, each coupled with two state-of-the-art coherent re- ceivers exploiting the two orthogonal polarization modes. The 56 receivers will be based on MMIC (Monolithic Microwave Integrated Circuits) technology, with HEMT (High Electron Mobility Transistor) ultra-low noise amplifiers (see e.g. Pospieszalski 1993; Kane et al. 1995). While even a moderate level of cryogenic cooling would improve the MMIC system noise, a passively cooled system greatly simplifies the design and can be operated for 
a duration limited only by spacecraft consumables (up to 5 years). Here we assume the HEMT detectors passively cooled at $T \simeq 100 \mathrm{~K}$, according to the present LFI baseline. The receivers will operate in four frequency bands $(31.5,53,90$, and $125 \mathrm{GHz})$, with the three lowest bands matching the COBE-DMR channels to facilitate the comparison of the results. Figure 2 shows a schematic of the LFI array geometry and the distribution of the four frequencies in the array. The number of channels at each frequency and their location have been chosen to optimize the sensitivity at 53 and $90 \mathrm{GHz}$, where CMB fluctuations are expected to dominate over any confusion foreground source, to minimize the effect of near-field distortions of the beams, and to provide consecutive observation of the same sky circles with more than one feed for internal crosscheck.

Fig. 2. Schematic of the beam footprints of the COBRAS/SAMBA Low Frequency Instrument (LFI) array. The circles represent the $30^{\prime}$ FWHM beams projected in the sky. The telescope focus corresponds to the $\left(0^{\circ}, 0^{\circ}\right)$ point in the plot. The most de-centered feed elements have projected beams $\sim 6.5^{\circ}$ away from the center of the field of view. The asymmetric geometry of the array is due to the presence of the HFI bolometer dewar which shares about half of the useful focal plane. $A, B, C$ and $D$ represent beams of 31.5, 53, 90 and $125 \mathrm{GHz}$ respectively. The beam footprints reproduce to first order the physical configuration of the feeds array (we have neglected in our simulations second-order effect due to beam distortion and asymmetries). The length scale on the top-right shows the corresponding physical feed-feed separation on the focal plane instrument

A receiver with bandwidth $\Delta \nu$ and effective system temperature $T_{\text {sys }}$ is limited by the statistics of thermal noise at a level $\delta T_{\text {rms }}=T_{\text {sys }} / \sqrt{\Delta \nu \tau}$, where $\tau$ is the inte- gration time. ${ }^{2}$ In the case of the LFI, if $N$ is the number of channels at the frequency $\nu$, the overall sensitivity of the array is improved by a factor $\sqrt{N}$ :

$$
\delta T_{\mathrm{rms}, \nu}=K_{\mathrm{r}} \frac{T_{\mathrm{sys}}}{\sqrt{\Delta \nu N \tau}},
$$

where the constant $K_{\mathrm{r}}$, of order of unity, depends on the type of radiometer. At present two receiver concepts are considered for the LFI: a differential concept, with continuous switching of the sky signal with the signal from a stable internal reference load (Bersanelli et al. 1995b), for which $K_{\mathrm{r}}=\sqrt{2}$; and a total-power concept, with $K_{\mathrm{r}}=1$. We will assume the latter case in this work. The values given in Table 1 correspond to performances in noise temperature and bandwidth currently obtained in prototype HEMT devices, or assume moderate extrapolation of the present performances at the higher frequencies (90 and $125 \mathrm{GHz})$.

The present baseline LFI angular resolution $\left(0.5^{\circ}\right.$ at all frequencies) has been assumed in this work. We have performed preliminary radiometric simulations of the global radiation pattern of the entire system (telescope, array feeds and radiation shield). They show good symmetry of the response, with acceptable levels of main-lobe and side-lobe distortions for all frequencies and for all locations in the focal plane. Analysis of the far-sidelobe rejection, shows that a rejection level of $\sim 65$ to $70 \mathrm{~dB}$ is achieved in the angular range up to $\sim 65^{\circ}$ around the main lobe, and a rejection level of $\sim 90$ to $100 \mathrm{~dB}$ for larger angles. The step between the two rejection levels is due to the presence of the radiation shield which is effective for large off-axis angles. Although the actual shape of the instrument angular response is somewhat asymmetric and depends on the frequency and location of the single feed in the focal plane array, the above numbers appear in general a realistic approximation for all cases. We have therefore modeled the instrumental angular response of each channel using the detailed shape of the main lobe resulting from the simulations with representative rejection levels at larger angles (see Fig. 3).

\section{Sensitivity and sky coverage}

\subsection{Algorithm description}

Using the instrumental parameters and scanning technique described in Sect. 2, we have evaluated the sky coverage and the sensitivity that can be achieved either

${ }^{2}$ Here we assume radiometric signals calibrated in terms of antenna temperature, defined as

$$
T_{\mathrm{A}} \equiv \frac{P}{k \Delta \nu}=\frac{x}{\left(\mathrm{e}^{x}-1\right)} T
$$

where $P$ is electromagnetic power intercepted by the antenna, $T$ is thermodynamic temperature, and $x \equiv h \nu / k T$. 
Fig. 3. Instrument angular response. We assume circularly symmetric beam patterns so $P$ is only dependent on the angular distance $\theta$ of the source from the beam center

from EM-L5 and from SE-L2. We assume that the spacecraft will be fixed in the Lagrangian points and neglect the second-order effect of orbital motion around them. This simplifying assumption does not change significantly any of the conclusions of this work. The simulation algorithm proceeds as follows. At a given time we orient the direction of the spin axis in the anti-sun direction. Then we consider the circle covered by the array beams when the satellite performs one revolution. The beams in the central row of the array will scan 676 pixels of $30^{\prime}$ size every rotation, with slightly different number of pixels for the beams in the adjacent rows. We over-sample the observed sky and divide the circle covered by each beam in 1800 elements, each defined by a direction $\left(\alpha_{i}, \delta_{i}\right)$. For each direction we need to evaluate the signal due to the relevant local sources. These are the Sun, the Earth, the Moon, and the external planets Mars, Jupiter, Saturn, Uranus, Neptune. Each of these sources is observed by the LFI channels as a signal of temperature

$$
\bar{T}_{j}=\frac{\int \mathrm{d} \Omega T_{j}(\theta, \phi) P(\theta, \phi)}{\int \mathrm{d} \Omega P(\theta, \phi)},
$$

where $T_{j}(\theta, \phi)$ is a function describing the radiometric temperature of the $j$-th source, and $P(\theta, \phi)$ is the instrument angular response. Our algorithm evaluates the cumulative signal, $T_{\mathrm{LS}}$, due to these local sources as:

$$
T_{\mathrm{LS}}=\sum_{j} \bar{T}_{j} \simeq \sum_{j} T_{j} \frac{\Omega_{j}}{\Omega_{\mathrm{B}}} P(\theta)
$$

where $\Omega_{\mathrm{B}} \equiv \int \mathrm{d} \Omega P(\theta, \phi)$, and the $\Omega_{j}$ 's are the solid angles of the relevant local sources. Because we assume circularly symmetric beam patterns, the instrumental angular response $P$ is only dependent on the angular distance $\theta$ of the source from the beam center (see Fig. 3 ).

The approximation in Eq. (3) is highly accurate for sources with angular dimension much less than the beam size: this is definitely true for all external planets (see Table 2). On the contrary, it is clearly incorrect for extended sources such as the Earth, the Moon and the Sun, with angular dimension comparable or larger than the beam size, when the sources are in main lobe. Nonetheless, we kept the simplifying assumption of Eq. (3), as we are only interested in an estimate of the contribution of these sources when they are away from the beam axis and contribute to the signal through the side- and back-lobes.

Once $T_{\mathrm{LS}}$ is computed for each $\left(\alpha_{i}, \delta_{i}\right)$, we evaluate the effect of solar system sources contamination by ignoring all those elements of the scan circle where $T_{\mathrm{LS}}$ exceeds a given threshold, $T_{\text {thre }}$. We choose as representative threshold values $T_{\text {thre }}=3.5,10$ and $100 \mu \mathrm{K}$, respectively. A threshold more restrictive than $3.5 \mu \mathrm{K}$ is meaningless: in fact, with the assumed beam pattern, the Sun and the Earth when they are both in the far lobes of the instrument at angles $\gtrsim 65^{\circ}$ from the spin axis contribute respectively $2.0 \mu \mathrm{K}$ and $1.2 \mu \mathrm{K}$ in L5 and 2.0 and $0.1 \mu \mathrm{K}$ in $\mathrm{L} 2$. The 10 and $100 \mu \mathrm{K}$ values define a range comparable to the cosmic fluctuations of interest. Note that the approach used here is conservative in the sense that the threshold cuts are based on the total signal $T_{\mathrm{LS}}$, while the observations are only sensitive to variations of $T_{\mathrm{LS}}$ as the instrument scans different portions of the sky. For localized, near-point-sources, however, we prefer this approach, since significant variations $\delta T_{j} / T_{j}$ might occur as 
Table 2. Minimum and maximum LFI temperature and angular diameter of local sources ${ }^{a, b}$

\begin{tabular}{lcccc}
\hline Source & $\begin{array}{c}T_{\min } \\
(\mu \mathrm{K})\end{array}$ & $\begin{array}{c}T_{\max } \\
(\mu \mathrm{K})\end{array}$ & $\begin{array}{c}\theta_{\min } \\
(\mathrm{deg})\end{array}$ & $\begin{array}{c}\theta_{\max } \\
(\mathrm{deg})\end{array}$ \\
& & & & 0.52 \\
Sun & 2.0 & $2.2,2.1$ & $0.48,0.11$ & $0.54,0.18$ \\
Moon & $\ll 1$ & $2.4 \times 10^{8}, \ll 1$ & $1.80,0.48$ & $2.04,0.48$ \\
Earth & $1.2,0.1$ & $3.0 \times 10^{8}, 0.1$ & & \\
& & & & \\
Mars & $\ll 1$ & $5.1 \times 10^{3}$ & $1.11 \times 10^{-3}$ & $3.84 \times 10^{-3}, 3.91 \times 10^{-3}$ \\
Jupiter & $\ll 1$ & $8.43 \times 10^{4}, 8.37 \times 10^{4}$ & $8.72 \times 10^{-3}$ & $1.27 \times 10^{-2}$ \\
Saturn & $\ll 1$ & $1.38 \times 10^{4}, 1.35 \times 10^{4}$ & $4.32 \times 10^{-3}$ & $5.34 \times 10^{-3}$ \\
Uranus & $\ll 1$ & $5.48 \times 10^{2}$ & $9.68 \times 10^{-4}$ & $1.07 \times 10^{-3}$ \\
Neptune & $\ll 1$ & $1.86 \times 10^{2}$ & $5.97 \times 10^{-4}$ & $6.38 \times 10^{-4}$ \\
& & & & \\
Total & $3.3,2.0$ & $3.0 \times 10^{8}, 8.4 \times 10^{4}$ & &
\end{tabular}

${ }^{a}$ When two values are shown, the first refers to EM-L5, the second to SE-L2.

${ }^{b}$ The SE-L2 values assume the spacecraft stable at the L2 point. More realistic scenarios (e.g. a Lissajous or halo orbit) would slightly change these values.

a result of angular variations in the beam pattern $P(\theta, \phi)$ at large angles. ${ }^{3}$

Our simulations take into account some details of the current design of the Low Frequency Instrument (LFI), i.e its performance, the number of feeds per frequency, and the physical displacement in the focal plane of the single feeds with respect to the optical focus (see Fig. 2). Following the mission baseline we assume that the telescope is moved (instantaneously) by $5^{\prime}$ every two hours. Here we neglect the effects of any possible drifts in the pointing direction or spin rate. Under this assumption, each feed describes the same sky-circle for two hours.

Finally, we divide the sky in $30^{\prime} \times 30^{\prime}$ pixels and evaluate the cumulative integration time spent on each pixel during one year mission. In order to do that we consider the pointing direction of each beam axis as sampled 1800 times during a spin. Each element $\left(\alpha_{i}, \delta_{i}\right.$, with $1 \leq i \leq 1800)$ is approximately $12^{\prime}$ in angular size, and corresponds to an elementary integration time $\tau_{o}=60$ $\mathrm{s} / 1800=33 \mathrm{~ms}$. (We refer to a pixel to indicate a $30^{\prime} \times 30^{\prime}$ angular unit, and to an element to indicate the oversampling $12^{\prime} \times 12^{\prime}$ elementary unit). The number of times, $N_{\text {obs }}^{(i)}$, that the pointing axis is sampled within the $i$-th element times $\tau_{o}$ is the total time spent on the $i$-th element. The total integration time to be attributed to a

\footnotetext{
${ }^{3}$ If a source with temperature $T$ crossed a $3 \mathrm{~dB}$ sidelobe feature as the beam scans the sky, this would result in $\delta T_{j} / T_{j} \sim 1$. At the extreme rejection levels required $(\sim-90 \mathrm{~dB})$ it appears unrealistic to achieve such accuracy in the determination of all $P(\theta, \phi)$ at large angles.
}

given $30^{\prime} \times 30^{\prime}$ pixel is then:

$$
\tau_{\mathrm{pix}}=\tau_{o} \times\left(\sum_{i} N_{\mathrm{obs}}^{(i)}\right)
$$

where the sum is extended to all the $12^{\prime} \times 12^{\prime}$ elements within a given $30^{\prime} \times 30^{\prime}$ sky pixel. The final sensitivity per pixel at a given frequency will be:

$$
\delta T_{\text {pix }}=K_{\mathrm{r}} \frac{T_{\text {sys }}}{\sqrt{\Delta \nu \sum_{k} \tau_{\text {pix }}^{(k)}}}
$$

where $\tau_{\text {pix }}^{(k)}$ is the integration time due to the $k$-th radiometer of the array at that frequency. This estimate is accurate as long as gain and offset long-term drifts can be removed from the measured data stream and residual $1 / f$ noise is negligible compared to the statistical noise. The receiver concept of the LFI and the scanning technique will be chosen to insure that these conditions will be met.

\subsection{Results}

Using the procedure described in the previous paragraph we have produced full-sky maps of sensitivity for both EM-L5 and SE-L2 (see Figs. 4a and 4b). Note that all the results presented here refer to one year mission. The ultimate sensitivity is therefore obtained accounting for a factor $\sqrt{N_{y}}$, where $N_{y}$ is the mission lifetime in years $(2$ to 5 for the LFI).

In order to quantify the visual impressions of the maps we produced a series of plots and histograms of selected quantities. A first quantitative comparison between EML5 and SE-L2 is given in Fig. 5, where we plot the fraction 
$f$ of the circle described by the beam for which $T_{\mathrm{LS}}<$ $T_{\text {thre, }}$ averaged over the number of channels at a given frequency. The reduction of useful observing time in EM$\mathrm{L} 5$, compared to SE-L2, is due to the periodic presence of the Earth and Moon near the field of view. It is evident that, observing from L5, a threshold $T_{\text {thre }}=3.5 \mu \mathrm{K}$ is very restrictive: only 34 per cent of the total observing time is useful for further data analysis. If we increase the threshold to $10 \mu \mathrm{K}, f$ has a mean value of $60 \%$ and for $T_{\text {thre }}=100 \mu \mathrm{K}, f$ has an average of $73 \%$. For comparison, observing from L2, $f$ is $>99 \%$ with any threshold $>5 \mu \mathrm{K}$.

In Fig. 6 we show histograms of the pixel sensitivity as defined in Eq. (5) for the $53 \mathrm{GHz}$ channel and for different thresholds. Figures $4 \mathrm{a}$ and $4 \mathrm{~b}$ show the full sky maps of pixel sensitivity at $53 \mathrm{GHz}$ and for $T_{\text {thre }}=10 \mu \mathrm{K}$ from EM-L5 and SE-L2. The results for the $90 \mathrm{GHz}$ channel are similar. The relevant statistical quantities are given in Table 3 both for EM-L5 and for SE-L2. Clearly for the SE-L2 case the dependence of the result on the threshold is much less important than in EM-L5. This is due to the fact than in SE-L2 only the planets contribute to $T_{\mathrm{LS}}$, and their contribution is much less important than that of the Earth or the Moon in EM-L5.

In the previous analysis, we lost any information about the coherence pattern in the map. In order to keep memory of it, we find the number of connected regions where the sensitivity is better than $T_{\mathrm{s}}$. As the pixel dimension is $30^{\prime} \times 30^{\prime}$ and the map is an equal area projection we can immediately evaluate the angular extension of the connected areas. From EM-L5 we expect to have $\sim 10$ connected $\left(10^{\circ} \times 10^{\circ}\right)$ regions around each of the two unobserved ecliptic polar caps with a sensitivity better than $10 \mu \mathrm{K}$. From SE-L2 this number increases up to 70 .

Note that our way of quoting the pixels sensitivity can be slightly misleading. In fact, when we increase the threshold $T_{\text {thre }}$, we increase the number of times that a given sky pixel has been observed, and then the sensitivity achieved in that pixel. However, for the same reason we also increase the contribution to the sky pixel due to solar system sources. So, a complementary information should be used, namely the mean contribution per pixel of the local sources: $\left\langle T_{\mathrm{LS}}\right\rangle=\sum_{m} T_{\mathrm{LS}}^{(m)} / N_{\text {obs }}$, where the sum is extended to all observations of a given pixel. Maps of this quantity are shown in Figs. 4c and 4d. It is clear that if the threshold is kept low, then $\left\langle T_{\mathrm{LS}}\right\rangle$ is small and very uniform over the sky. If the threshold is increased too much then the contribution from local sources becomes dominant, and the mean temperature per pixel is not uniform over the sky. A visual impression of sensitivity maps and of $\left\langle T_{\mathrm{LS}}\right\rangle$ maps shows that a threshold $\approx 10 \mu \mathrm{K}$ is just about right in order to maximize the sky coverage and to minimize the residuals of the local sources emission. This can be again quantified in terms of histograms (see Fig. 6 and Tables $3 \mathrm{a}$ and $3 \mathrm{~b})$.
Our radiometric simulations indicate a beam pattern with a typical response less than $-90 \mathrm{~dB}$ for $\theta \gtrsim 65^{\circ}$. In the case SE-L2 the main local sources (Sun, Earth and Moon) constantly fall in this maximum rejection region. This is reflected in the fact that the residual $\left\langle T_{\mathrm{LS}}\right\rangle$ (Table $3 \mathrm{~b}$ ) is nearly constant for different thresholds. If the beam rejection in this region were significantly less efficient than expected, then the average contribution of local sources would proportionally increase. So, if the assumed response at $\theta \gtrsim 65^{\circ}$ were wrong by a factor of ten it would be enough to increase $T_{\text {thre }}$ by the same factor. The weak dependence of the sensitivity shown in Fig. 6 with $T_{\text {thre }}$, at least in the case SE-L2, suggests that our estimates are quite robust. Moreover, as mentioned in Sect. 3.1, the presence of a term $T_{\mathrm{LS}}$ during a scan does not necessarily preclude the observations, as long as $T_{\mathrm{LS}}$ is constant. In particular the Sun (the main local source from SE-L2) will be constantly seen through the same beam direction $(\theta \simeq$ $110^{\circ}$ ) during normal anti-sun observation, thus adding a pure baseline to the signal. A complete analysis of the beam reconstruction during orbit and transfer phase will permit a more refined assessment of the mission sensitivity to potential underestimates of the off-axis radiation and irregularities in the beam patterns. This is beyond the purpose of this paper, but it is being undertaken as part of our mission study effort.

\subsection{Galactic emission}

The results in the previous paragraph do not take into account constraints arising from the presence of diffuse microwave emission from our Galaxy. In this section we quantify these constraints and we study how these affect the previous results. To this purpose we set up a crude model of Galactic emission arising from the combination of synchrotron and free-free radiation. We neglect thermal radiation from interstellar dust, which is fainter at the considered wavelengths, although it is expected to contribute at the higher LFI frequencies. We stress the fact that this model is not accurate enough to subtract a Galactic component from the detected signals. Internal fits on the data, taking advantage of the multi-band nature of the experiment, will be much more effective for the removal of foreground radiation. We will use our approximate Galactic model mainly to see how much the useful sky coverage is reduced by the presence of intense foreground radiation near the Galactic plane.

In general, the increase in the thermodynamic temperature due to the presence of Galactic radiation, $\Delta T_{\text {Galaxy }}$, at observing frequency $\nu$, can be written as

$$
\frac{\Delta T_{\text {Galaxy }}(\nu)}{T_{\mathrm{CMB}}}=\frac{\left(\mathrm{e}^{x}-1\right)}{x \mathrm{e}^{x}} \frac{F_{\text {Galaxy }}(\nu)}{F_{\mathrm{CMB}}},
$$

where $x \equiv h \nu / k T_{\mathrm{CMB}}, F_{\text {Galaxy }}$ is the flux of the Galactic radiation, and $F_{\mathrm{CMB}}$ is the Planck function with $T=$ $T_{\mathrm{CMB}}=2.726 \mathrm{~K}$ (Mather et al. 1994). 
Fig. 4. Full sky map in equatorial coordinates of the sensitivity reached after an year of observations at 53 GHz, from EM-L5 (panel a) and from SE-L2 (panel b), assuming a rejection threshold $T_{\text {thre }}=10 \mu \mathrm{K}$ (see text). Full sky map in equatorial coordinates of the mean temperature contribution (per pixel) of the solar system sources at $53 \mathrm{GHz}$, from EM-L5 (panel c) and from SE-L2 (panel d), assuming a rejection threshold $T_{\text {thre }}=10 \mu \mathrm{K}$ (see text). Synthetic map in equatorial coordinates of galactic synchrotron and free-free emission (panel e) at $53 \mathrm{GHz}$ (see text)

The synthetic synchrotron emission at the frequencies of interest has been obtained scaling the Haslam (1982) map at $408 \mathrm{MHz}$. For our purposes the poor accuracy $(5-10 \%)$ and the presence of instrumental features at low levels are not an important problem. Assuming an antenna temperature spectral index $\left(T_{\mathrm{A}} \propto \nu^{\gamma}\right)$ of $\gamma=-2.75$ (see e.g. Banday \& Wolfendale 1990) from Eq. (6) we derive the scaling law

$$
\Delta T_{\text {sync }}(\nu)=T_{\mathrm{A}}(408 \mathrm{MHz}) \frac{\left(\mathrm{e}^{x}-1\right)^{2}}{x^{2} \mathrm{e}^{x}}\left(\frac{\nu}{408 \mathrm{MHz}}\right)^{-2.75} .
$$

The scaling factors obtained in this way are $6.6,1.7,0.44$ and $0.21 \mu \mathrm{K}_{\mathrm{CMB}} / \mathrm{K}_{408 \mathrm{MHz}}$ at $31.5,53,90$ and $125 \mathrm{GHz}$, respectively. The resulting synchrotron maps have been interpolated in our 30 arcmin grid and convolved with the beam profile of Fig. 3 .
The synthetic free-free emission has been obtained from a catalogue of 881 microwave fluxes of HII regions in the Galactic plane (Witebsky 1988). Here the scaling law is

$$
\Delta T_{\mathrm{ff}}(\nu)=T_{\mathrm{CMB}} \frac{F_{\mathrm{ff}}\left(\nu_{1}\right)}{F_{\mathrm{CMB}}}\left(\frac{\nu}{\nu_{1}}\right)^{-0.1} \frac{\left(\mathrm{e}^{x}-1\right)}{x \mathrm{e}^{x}} \frac{1}{\Omega_{\mathrm{B}}}
$$

where $F\left(\nu_{1}\right)$ is the flux from the HII region, $\nu_{1}$ is the frequency in the catalogue, and $\Omega_{\mathrm{B}}$ is the beam-size of the telescope. Again, the synthetic free-free map has been obtained by convolving the flux from all the HII regions with the beam profile of Fig. 3. This approach does not attempt to model faint features present in high Galactic latitude free-free emission (see Reynolds 1992).

An important concern with Galactic plane emission is that its presence in the sidelobes may be important even 
Fig. 5. The fraction $f$ of the circle described by the beam for which $T_{\mathrm{LS}}<T_{\text {thre }}$, averaged over the number of feeds at 53 GHz. Panels a)-c) refer to EM-L5: $T_{\text {thre }}$ is assumed to be $100 \mu \mathrm{K}, 10 \mu \mathrm{K}, 3.5 \mu \mathrm{K}$, respectively. Panels d)-f) refer to SE-L2: $T_{\text {thre }}$ is again $100 \mu \mathrm{K}, 10 \mu \mathrm{K}, 3.5 \mu \mathrm{K}$, respectively 
Fig. 6. Pixel sensitivity distributions at $53 \mathrm{GHz}$ (left panels) achievable from EM-L5 (dotted lines) and SE-L2 (heavy continuous

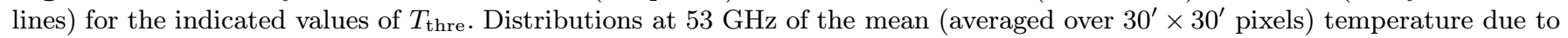
solar system sources (right panels) achievable from EM-L5 (dotted lines) and SE-L2 (heavy continuous lines) for the indicated values of $T_{\text {thre }}$ 
Table 3. a) Summary of results for EM-L5 (First Year Mission)

\begin{tabular}{cccccccc}
\hline $\begin{array}{c}\text { Band } \\
(\mathrm{GHz})\end{array}$ & $\begin{array}{c}T_{\text {thre }} \\
(\mu \mathrm{K})\end{array}$ & $\begin{array}{c}f \\
(\%)\end{array}$ & $\begin{array}{c}\text { Coverage } \\
(\%)\end{array}$ & $\begin{array}{c}\left\langle\delta T_{\text {pix }}\right\rangle \\
(\mu \mathrm{K})\end{array}$ & $\begin{array}{c}\text { rms } \\
(\mu \mathrm{K})\end{array}$ & $\begin{array}{c}\left\langle T_{\mathrm{LS}}\right\rangle \\
(\mu \mathrm{K})\end{array}$ & $\begin{array}{c}\text { rms } \\
(\mu \mathrm{K})\end{array}$ \\
\hline 31.5 & 3.5 & 34 & 69 & 31.2 & 18.2 & 3.4 & 0.05 \\
& 10 & 60 & 88 & 25.8 & 13.2 & 3.5 & 0.44 \\
& 100 & 73 & 94 & 23.1 & 10.6 & 6.5 & 6.45 \\
& & & & & & & \\
53 & 3.5 & 34 & 82 & 21.6 & 17.1 & 3.4 & 0.04 \\
& 10 & 60 & 96 & 15.9 & 10.1 & 3.6 & 0.45 \\
& 100 & 73 & 97 & 13.1 & 5.7 & 6.4 & 3.97 \\
& & & & & & & \\
90 & 3.5 & 34 & 89 & 28.4 & 24.1 & 3.4 & 0.03 \\
& 10 & 60 & 97 & 18.9 & 9.2 & 3.6 & 0.45 \\
& 100 & 73 & 98 & 16.3 & 6.8 & 6.1 & 2.91 \\
& & & & & & & \\
125 & 3.5 & 34 & 87 & 68.2 & 46.6 & 3.4 & 0.04 \\
& 10 & 60 & 97 & 40.9 & 19.0 & 3.6 & 0.44 \\
& 100 & 73 & 98 & 16.5 & 15.3 & 6.0 & 2.96 \\
\hline
\end{tabular}

Table 3. b) Summary of results for SE-L2 (First Year Mission)

\begin{tabular}{cccccccc}
\hline $\begin{array}{c}\text { Band } \\
(\mathrm{GHz})\end{array}$ & $\begin{array}{c}T_{\text {thre }} \\
(\mu \mathrm{K})\end{array}$ & $\begin{array}{c}f \\
(\%)\end{array}$ & $\begin{array}{c}\text { Coverage } \\
(\%)\end{array}$ & $\begin{array}{c}\left\langle\delta T_{\text {pix }}\right\rangle \\
(\mu \mathrm{K})\end{array}$ & $\begin{array}{c}\mathrm{rms} \\
(\mu \mathrm{K})\end{array}$ & $\begin{array}{c}\left\langle T_{\mathrm{LS}}\right\rangle \\
(\mu \mathrm{K})\end{array}$ & $\begin{array}{c}\text { rms } \\
(\mu \mathrm{K})\end{array}$ \\
\hline 31.5 & 3.5 & 98.6 & 95 & 17.6 & 5.6 & 2.2 & 0.1 \\
& 10 & 99.7 & 95 & 17.5 & 5.2 & 2.2 & 0.5 \\
& 100 & 99.9 & 96 & 17.4 & 5.0 & 2.3 & 1.4 \\
& & & & & & & \\
53 & 3.5 & 98.6 & 97 & 10.2 & 4.4 & 2.2 & 0.1 \\
& 10 & 99.6 & 97 & 10.1 & 4.0 & 2.2 & 0.5 \\
& 100 & 99.9 & 97 & 10.1 & 3.8 & 2.3 & 1.5 \\
& & & & & & & \\
90 & 3.5 & 98.5 & 97 & 13.1 & 6.4 & 2.2 & 0.1 \\
& 10 & 99.6 & 98 & 13.0 & 6.0 & 2.2 & 0.5 \\
& 100 & 99.9 & 98 & 12.9 & 5.6 & 2.3 & 1.5 \\
& & & & & & & \\
125 & 3.5 & 98.5 & 97 & 33.0 & 13.6 & 2.2 & 0.1 \\
& 10 & 99.6 & 97 & 32.7 & 12.7 & 2.2 & 0.5 \\
& 100 & 99.9 & 98 & 32.6 & 12.1 & 2.3 & 1.6 \\
\hline
\end{tabular}

when the instrument is observing high Galactic latitude regions. In fact we find that the Galactic plane emission belt fills a considerable solid angle $(\sim 2 \mathrm{sr})$, with an average level of $\sim 100 \mu \mathrm{K}$ at $53 \mathrm{GHz}$. This means that with a typical rejection level of $-70 \mathrm{~dB}$ at $30^{\circ}$ off axis, the Galactic plane can produce signals at the $3 \mu \mathrm{K}$ level even when the telescope is observing at Galactic latitudes of the order of $30^{\circ}$. Obviously this effect is more evident at $31.5 \mathrm{GHz}$. By comparing unconvolved and convolved maps we find that the contribution of the sidelobes is comparable to the intrinsic emission of the Galaxy at high Galactic latitudes.
The resulting synthetic map of the Galaxy is shown in Fig. 4e. This is dominated at high fluxes by HII regions, mostly close to the Galactic plane. High Galactic latitude features (e.g. the Galactic Spur etc.) are due to synchrotron emission, at remarkably lower levels. The influence of HII regions in the sidelobes of the beam is not negligible even at Galactic latitudes of order 20 degrees.

In Fig. 7 we plot the average and the pixel to pixel rms of the synthetic Galactic maps as a function of the cut latitude. The behavior of these quantities is dominated by high level signal spikes due to HII regions. As the cut latitude increases, the weight of the relatively few spikes decreases with respect to the weight of low 
level diffuse emission fluctuations, and the rms decreases. The characteristic step at $b=20^{\circ}$ is due to cut of the last important HII regions. The second step present at $b=32^{\circ}$ in the full sky maps is due to the presence of HII sources in the two high latitude regions unobserved in the COBRAS/SAMBA scan.

We conclude that a $20^{\circ}$ Galactic cut is necessary and sufficient to perform high sensitive measurements with negligible contributions from the Galaxy.

Finally, we note that the signal from the Galactic plane can be used for a relative calibration of the instrument, i.e., an in-flight monitoring of the stability in time of the detectors gain. This will be particularly efficient at the higher bolometric bands, where the Galaxy has more power. Frequent absolute calibration (i.e., measurements of the calibration constant) will be insured by the CMB dipole signal, with occasional cross-checks provided by external planets falling in the field of view of the instruments. Details of the calibration technique and expected accuracies will be discussed in a forthcoming paper.

\section{Conclusions}

The main results of our full-sky simulations are summarized in Tables $3 \mathrm{a}$ and $3 \mathrm{~b}$. The following main points emerge from this work.

1. The instrumental sensitivity expected in the next generation of radiometers is sufficient to produce extended maps of the CMB anisotropies at sub-degree angular scales, with sensitivity per pixel of order $\sim 10 \mu \mathrm{K}$. This is shown for the particular case of COBRAS/SAMBA, in the sky maps of Figs. $4 \mathrm{a}$ and $4 \mathrm{~b}$ and in Fig. 6 and will allow a powerful discrimination among different cosmological models. The mean sensitivity of pixels with a sensitivity better then $10 \mu \mathrm{K}$ is $\sim 8 \mu \mathrm{K}$ from both EM-L5 and SE-L2, but the total sky coverage of these regions is $\lesssim 3 \%, \lesssim 20 \%, \lesssim 11 \%$ (at $31.5,53$ and 90 GHz respectively) from EM-L5 and 3\%, 36\% and 14\% from SE-L2.

2. A very important part in the optimization of the observations is played by the choice of orbit. A far-Earth orbit is needed if one wishes to drastically reduce the off-axis radiation from the Earth. However, our comparison between SE-L2 and EM-L5 shows that even from an orbit at lunar distance the observation time can be substantially reduced due to the Sun-EarthMoon-Spacecraft geometry (Fig. 5). Moving from EML2 to SE-L2, the fraction of useful integration time increases very significantly. Using as a reference the threshold $T_{\text {thre }}=10 \mu \mathrm{K}$, for which the residual contamination from local sources is still acceptable, the useful integration time increases from $60 \%$ to more than $99 \%$. Even assuming the very conservative cut $T_{\text {thre }}=3.5 \mu \mathrm{K}$ we find that from SE-L2 the "good" data would be $98.5 \%$ of the total, while from EM-L5 it would drop to about $34 \%$. This is the main cause of the contrast between the two cases shown by the sensitivity histograms (Fig. 6).

3 . The great advantage of the L2 orbit is the fact that the Earth and the Moon are always at large aspect angles when keeping the spin axis constantly near antisun. Our simulations assumed the same simple baseline scanning technique for both EM-L2 and SE-L2. Clearly, one could relax this assumption and construct a more complicated scanning plan, involving periodic re-pointing of the spin axis, in order to optimize avoidance of Earth and Moon from EM-L5. This, however, would have a serious impact on the thermal configuration of the spacecraft since a significant change in the solar aspect angle would introduce a spin-synchronous modulation affecting the stringent thermal stability requirements of both LFI and HFI.

4. The sensitivity distribution over the sky for the baseline scan strategy is shown in Figs. $4 \mathrm{a}$ and $4 \mathrm{~b}$. As mentioned in Sect. 3.2, we find that mapping from SE-L2 will yield a large number of extended, high-sensitivity, connected regions. This has a number of consequences for the scientific objectives one can derive from the observations. In particular, the discrimination between Gaussian or non-Gaussian (e.g. produced by topological defects such as cosmic strings or textures) statistics of the primordial perturbations require extended, connected regions of CMB mapping at high sensitivity (e.g. Bennett et al. 1992). Small corrections $\left(\stackrel{\sim}{\sim} 10^{\circ}\right)$ of the spin axis direction not affecting the total-shadow condition (and thus the thermal stability of the instruments) should be planned in order to optimize the sensitivity distribution according to the scientific goals.

5. A non-cosmological emission that cannot be eliminated is the radiation of Galactic origin. Although Galactic radiation is highly concentrated in the Galactic plane, it prevents accurate CMB observations in a significant fraction of the sky. Moreover, Galactic radiation in the sidelobes of the instrument can produce non-negligible effects. We find that with a rejection of about $-70 \mathrm{~dB}$ the signal from the Galactic plane is reduced to few $\mu \mathrm{K}$ level. Residual effects would be spin-synchronous within a measurement set (120 min), and could affect the low multipole statistics. However, the same pixels will be observed with different phases with respect to the Galactic plane during the mission, so that possible residual effect will average out to some extent, and in principle they could even be traced and corrected.

Although the details of our simulations are based on the COBRAS/SAMBA characteristics and mission study, the general conclusions apply to other similar proposed mission, or can be used for a quantitative comparison. In particular, it clearly emerges from our study that the choice of the SE-L2 orbit (or possibly other heliocentric 
Fig. 7. Mean (continuous lines) and pixel to pixel rms (dotted lines) of the Galactic contributions (HII regions and Synchrotron emission) as a function of the cut in Galactic latitude, $b$. In the right panels we have excluded the contribution from the two small unobserved ecliptic polar caps 
orbits allowing complete avoidance of the Earth and the Moon in anti-sun configuration) is highly recommended for a mission aiming to a sub-degree resolution imaging of the CMB anisotropies. Such goal is at reach and will generate an extraordinary new impulse to cosmology and astrophysics.

\section{References}

Banday A.J., Wolfendale A.W., 1990, MNRAS 245, 182

Bennett C.L., et al., 1992, ApJ 391, 466

Bennett C.L., Stebbins A., Bouchet F.R., 1992, ApJ 399, L5

Bersanelli, et al., 1995a, ApJ 448, 8

Bersanelli M., et al., 1995b, TESRE Int. Rep. No. 177/1995

Bond J.R., Crittenden R., Davis R.L., Efstathiou G., Steinhardt P.J., 1994, Phys. Rev. Lett. 72, 13

Brandt W.N., Lawrence C.N., Readhead A.C.S., Pakianathan J.N., Fiola T.M., 1994, ApJ 424, 1

Cheng E.S., et al., 1994, ApJ 422, L37

Church S., 1995, MNRAS 272, 551

Clapp A.C., et al., 1994, ApJ 433, L57

Coulson D., Ferreira P., Graham P., Turok N., 1994, Nat 368, 27

Crittenden R., Bond J.R., Davis R.L., Efstathiou G., Steinhardt P., 1993, Phys. Rev. Lett. 71, 324

Danese L., Toffolatti L., Franceschini A., Bersanelli M., Mandolesi N., 1996, Astroph. Lett Comm. 33, 257

De Bernardis P., et al., 1994, ApJ 422, L33

De Pater I., Matisse S.T., 1985, Icarus 62, 143

De Pater I., 1990, Ann. Rev. Astron. Astrophys. 28, 347

Devlin M.J., et al., 1994, ApJ 430, L1

Dragovan M., et al., 1994, ApJ 427, L67
Farquhar R.W., Dunham W.D., 1990, Observatories in Earth Orbit and Beyond. In: Kondo Y. (ed.). Kluver, p. 391

Gundersen J.O., et al., 1995, ApJ 443, L57

Haslam C.G.T., et al., 1982, A\&AS 47, 1

Kamionkowsky M., Spergel D.N., Sugiyama N., 1994, ApJ 426, L57

Kane B., Weinreb S., Fisher E., Byer N., 1995, MTT-S, Orlando, 1995 (preprint)

Kogut A., et al., 1993, ApJ 419, 1

Lubin P., 1995, Astroph. Lett. \& Comm. 32, 257

Mandolesi N., et al., 1993, COBRAS, proposal for ESA-M3

Mandolesi N., et al., 1995a, Planet. Space Sci. (in press)

Mandolesi N., et al., 1995b, Astroph. Lett \& Comm. 32, 309

Mather J.C., et al., 1994, ApJ 420, 439

Netterfield C.B., Jarosik N., Page L., Wilkinson D., Wollack E., 1995, ApJ 445, L69

Pospieszalski M.W., 1993, 23rd EuMC, p. 73

Puget J.L., et al., 1993, SAMBA, proposal for ESA-M3

Readhead A.C.S., Lawrence C.R., 1992, ARA\&A 30, 653

Reynolds R.J., 1992, ApJ Lett. 392, L35

Schuster J., et al., 1993, ApJ 412, L47

Smoot G.F., et al., 1992, ApJ 396, L1

Smoot G.F., et al., 1995, Astroph. Lett \& Comm. 32, 309

Toffolatti L., et al., 1995, Astrophys. Lett. \& Comm. 32, 125

Ulich B.L., et al., 1980, IEEE Trans. Antennas Propagat., AP-28, 367

Ulich B.L., 1981, AJ 86, 1619

Vittorio N., Meinhold P., Muciaccia P.F., Lubin P., Silk J., 1991, ApJ 372, L1

White M., Scott D., Silk J., 1994, ARAA 32, 319

Witebsky C., 1988 (private comunication)

Wollack E.J., et al., 1993, ApJ 419, L49 